\title{
Blochemical monitoring of genetic collections of strawberry in NCFSCHVW and CEBS
}

\author{
T.G. Prichko ${ }^{1, *}$, M.G. Germanova ${ }^{1}$, and V.N. Podorozhniy ${ }^{2}$ \\ ${ }^{1}$ FSBSI North Caucasus Federal Scientific Center of Horticulture, Viticulture, Winemaking (FSBSI \\ NCFSCHVW), st.. 40th let Pobedy, 39, Krasnodar, Russia \\ ${ }^{2}$ Russian Institute of Plant Genetic Resources named after N.I. Vavilova "(CEBS), st. Vavilova, 12, \\ Krymsk, Russia
}

\begin{abstract}
The article presents the generalized results of long-term research (2012-2020) of the assessment of the chemical composition of strawberries of 90 varieties of various ecological and geographical origin, growing in the genetic collections of NCFSCHVW and CEBS (branch of VIR). The advantage of varieties of Italian selection, as well as Russian (NCFSCHVW, CEBS) is the increased content of soluble solids and sugars. The varieties obtained in France and Russia are characterized by a high level of accumulation of vitamin $\mathrm{C}$. The largest amount of vitamin $\mathrm{P}$ is contained in strawberries of American varieties. According to the results of a study of five groups of strawberry varieties of different ripening periods, it was found that varieties of an early ripening period accumulate less soluble solids and sugars, and late-ripening ones stand out with a high content of soluble solids, sugars, vitamins $\mathrm{C}$ and $\mathrm{P}$. Highlighted varieties that are the sources of high soluble solids content $(9-9.9 \%)$, sugars $(6.8-$ $7.5 \%)$, acids (1.0-1.28\%), pectin substances $(0.7-0.79 \%)$, vitamin C ( $65.1-$ $74.5 \mathrm{mg} / 100 \mathrm{~g})$, vitamin P (90.0-120.1 mg / $100 \mathrm{~g})$ and anthocyanins (92.0-127.0 mg / $100 \mathrm{~g})$.
\end{abstract}

\section{Introduction}

Strawberry (Fragaria $\times$ ananassa Duch.) is a crop widespread in many natural and climatic zones of the globe, due to its early maturity, early maturity, high productivity, and adaptability to cultivation. Along with these economically valuable traits, the chemical composition of the fruits of garden strawberries is important, which determines their taste, aroma and texture, as well as nutritional properties and therapeutic and prophylactic qualities. Strawberries contain sugar $(4.6-8.6 \%)$, organic acids $(0.50-1.80 \%)$, pectin $(0.48$ $1.6 \%$ ), fiber (3-4\%), vitamin C (31.8-121.9 mg / $100 \mathrm{~g})$, vitamin P (50-200 mg / $100 \mathrm{~g})$, vitamin E $(0.54 \mathrm{mg} / 100 \mathrm{~g})$, anthocyanins $(37.8-105.1 \mathrm{mg} / 100 \mathrm{~g})$, minerals: potassium $126-161 \mathrm{mg} / 100 \mathrm{~g}$, calcium - 30-40 mg / $100 \mathrm{~g}$, magnesium - $18-22 \mathrm{mg} / 100 \mathrm{~g}$, iron $900-1200 \mu \mathrm{g} / 100 \mathrm{~g})[1-6]$.

The renewal of the assortment of strawberries in the North Caucasus region of Russia is carried out through the creation and introduction of new varieties of domestic selection, as well as introduced ones. The aim of the study is to assess the chemical composition of 
berries of various varieties of garden strawberries in the genetic collections of the culture growing in the Krasnodar Territory to isolate the sources of valuable traits used in breeding to improve the chemical composition.

\section{Materials and methods}

The studies were carried out in 2012-2020 in the laboratory for storage and processing of fruits and berries of the NCFSCHVW and the laboratory of biochemistry of the Crimean EBS - a branch of VIR in accordance with the "Program and methodology for the study of varieties of fruit, berry and nut crops" and "Program of the North Caucasus Center for the selection of fruit, berry, flower and ornamental crops and grapes for the period up to 2030 "' $[7,8]$.

The objects of the research were berries of 90 varieties of strawberries grown in the central and foothill zones of the Krasnodar Territory, including 10 new varieties of the NCFSCHVW selection and 5 varieties of the Crimean EBS selection. In the course of the research in strawberries, the following was determined: soluble solids - according to GOST ISO 2173-2013 with a digital refractometer PAL-3 (ATAGO); common sugars - by a photometric method based on the interaction of the carbonyl groups of sugars in an alkaline medium with ferrocyanide potassium and measuring the optical density of the resulting solution on a KFK-3-01 photoelectric colorimeter in accordance with GOST 8756.13-87; titratable acids - by titration in the presence of phenolphthalein indicator according to GOST ISO 750-2013; vitamin C - by the fluorometric method by interaction with ophenylenediamine with the formation of a fluorescent product according to M 04-07-2010 on "Fluorate-02"; vitamin P - by the colorimetric method with a vanillin reagent on the KFK-3-01 photoelectric colorimeter; pectin substances - by the carbazole method modified by E.V. Sapozhnikova, based on the interaction of carbazole with galacturonic acid. The experimental data obtained were processed by the methods of mathematical statistics using the analysis of variance in the Microsoft Office Excel program.

\section{Results and discussion}

The soluble dry matter of strawberries, the amount of which varied from 7.2 to $9.9 \%$, is represented mainly by sugars (5.4-7.8\%). The following varieties are distinguished by an increased content of soluble solids and sugars (from 9.0\% to 9.9\%): Darselect, Vystavochnaya, Ruby pendant, Molling Pegasus, Polka, Eris, Clery, Belrubi, Alaya, Cheburashka, Kamarosa, Elizabeth II, Gera and others (table 1).

Table 1. Strawberry varieties - sources of macro- and micronutrients.

\begin{tabular}{|c|l|}
\hline Indicators & \multicolumn{1}{|c|}{ Varieties } \\
\hline Soluble solids, & Darselect *, Vystavochnaya, Ruby \\
& pendant, Molling Pegasus, Shelf, Eris, \\
& Clery, Belrubi, Alaya, Cheburashka, \\
& Kamarosa, Elizabeth II, Gera, Vesper, \\
& Rusanovka, Zenga-Zengana \\
\hline Acids, $>1 \%$ & Nelly *, Belrubi, Gera, Vesper, Janie, \\
& Trubodur, Ruby pendant, \\
& Vystavochnaya, Cardinal, Symphony, \\
& Kemiya, Sunrise, Alaya, Martian, \\
& Cheburashka, Rusanovka, Presvyata, \\
& Alba, Alina, Teynira \\
\hline Pectin substances, & Clery *, Kemiya, Alba, Alaya, \\
& Elsanta, Arosa, Vystavochnaya, \\
& Bogota, Camarosa, Cardinal, Janie, \\
\hline
\end{tabular}




\begin{tabular}{|l|l|}
\hline & Marmolada, Honey \\
\hline Vitamin C,>65 mg / 100 g & Kemiya *, Vystavochnaya, Desna, \\
& Alaya, Gera, Sunrise, Elizabeth II, \\
& Alina, Florence, Primela, Darselect, \\
& Taira, Molling Pandora, Molling \\
& Pegasus, Cambridge favorite, Belrubi, \\
& Janie, Zenkora, Rusanovka, Presvyata, \\
& Holiday \\
\hline Vitamin P,>90 mg / 100 g & Selva *, Yuzhanka, Vystavochnaya, \\
& Arosa, Darselect, Evyu-2, Florence, \\
& Honey, Camarosa, Presvyata, Roxana, \\
& Holiday, Molling Pandora, Elizabeth \\
& II, Cambridge favorite, Maya, Syria, \\
& Karina, Gera, Daughter of the purple, \\
& Elsanta, Dukat \\
\hline Anthocyanins, $>90, \mathrm{mg} / 100 \mathrm{~g}$ & Belrubi *, Cardinal, Yuzhanka, \\
& Vystavochnaya, Sunrise, Vesper, \\
& Zenkora, Trubodur, Daughter of the \\
& purple, Martian, Zenga-Zengana, \\
& Fireworks, Kent, Rusanovka, Teinira, \\
& Kama, Dukat, Desna, Presvyata, \\
& Honey, Maryshka \\
\hline
\end{tabular}

* - the list of varieties is presented as the quantitative content of nutrients decreases

Depending on the variety, the acid content in strawberries can differ twofold - from $0.67 \%$ (Eros variety) to $1.28 \%$ (Nelly variety). High acidity, more than $1 \%$, is noted in the varieties such as Nelly, Belrubi, Gera, Vesper, Jani, Trubodur, Ruby pendant, Vystavochnaya, Cardinal, Symphony, Kemiya and others.

Strawberries are a rich source of vitamin $\mathrm{C}$, the content of which varies depending on varietal characteristics from $36.3 \mathrm{mg} / 100 \mathrm{~g}$ (Selva variety) to $74.6 \mathrm{mg} / 100 \mathrm{~g}$ (Kemiya, Exhibition). The content of vitamin $\mathrm{C}$ in the berries of the studied varieties is comparable with the data of a number of Russian and foreign authors. So, according to I.M. Skurikhin. [9] the average amount of vitamin C is $60.0 \mathrm{mg} / 100 \mathrm{~g}$; according to Giampieri F. - 58.8 $\mathrm{mg} / 100 \mathrm{~g}$ [10]. According to the research Lukyanchuk I.V. in Central Russia, the average vitamin C content in 73 strawberry varieties is $68.5 \mathrm{mg} / 100 \mathrm{~g}$ [11]. The following varieties are of particular value in terms of vitamin C content: Kemiya, Vystavochnaya, Desna, Alaya, Gera, Voskhod, Elizabeth II, Alina, Florence, Primela, Darselect, Taira and others, accumulating more than $65 \mathrm{mg} / 100 \mathrm{~g}$.

Typical differences in strawberry varieties are manifested in the quantitative content of vitamin P, while the variation ranges from $58.3 \mathrm{mg} / 100 \mathrm{~g}$ (Jani variety) to $122.1 \mathrm{mg} / 100 \mathrm{~g}$ (Selva variety), which is lower compared to the indicators for Central Russia (40-352 mg / $100 \mathrm{~g}$ ) [11]. It is worth highlighting the varieties such as Selva, Yuzhanka, Vystavochnaya, Arosa, Darselect, Evyu-2, Florence, Honey, Camarosa, Blessed, Roxana, Holiday, Molling Pandora, Elizabeth II, Cambridge favorite, Maya, Syria, Karina, Gera, Purple's daughter, Elsanta, Ducat with a vitamin P content of more than $90 \mathrm{mg}$ per $100 \mathrm{~g}$ of strawberries.

Variations in the content of anthocyanins, which determine the color of berries, were set from 60.0 (variety Jani) to $127.0 \mathrm{mg} / 100 \mathrm{~g}$ (variety Belrubi). It has been noted that berries grown in Central Russia (10.5-171.6 mg / $100 \mathrm{~g}$ ) [11] and in the southern Urals (25.0-95.3 $\mathrm{mg} / 100 \mathrm{~g}$ ) [12] are less colored. Intensely colored strawberries contain more than $90 \mathrm{mg} /$ $100 \mathrm{~g}$ of anthocyanins: Belrubi, Cardinal, Yuzhanka, Exhibition, Voskhod, Vesper, Zenkora, Trubodur, Daughter of the purple, Martian, Zenga-Zengana, Fireworks, Kent, Rusanovka, Teinira, Kama, Dukat, Desna and others.

An important advantage of strawberries is the presence of pectin substances in berries in an amount from 0.52 to $0.79 \%$, represented by protopectin and soluble pectin, which is comparable with the data for the Republic of Belarus (0.48-0.84\%) [13]. According to M.Yu. Akimov. berries grown in Central Russia contain more pectin substances (average 
$0.98 \%$ ) [3]. The varieties with a high pectin content have been identified: Clery $(0.79 \%)$, Kemiya $(0.77 \%)$, Alba $(0.75 \%)$, Alaya $(0.74 \%)$, Elsanta $(0.73 \%)$, Arosa $(0,72 \%)$, Camarosa $(0.72 \%)$, Vystavochnaya $(0.72 \%)$, Bogota $(0.71 \%)$, Cardinal $(0.71 \%)$.

It is well known that varieties of different ecological and geographical origin do not equally realize their potential when grown in different soil and climatic conditions [14-16]. Thus, when grown under the conditions of the Krasnodar Territory, the greatest accumulation of soluble solids and sugars in berries was noted in the varieties of Italian selection, as well as the selection of NCFSCHVW and Crimean EBS - 8.6\% with a low coefficient of variation ( $\mathrm{V}=6.4$ and $7.4 \%$ ) (table 2$)$.

Table 2. The chemical composition of strawberries of various ecological and geographical origin, grown in the south of Russia.

\begin{tabular}{|c|c|c|c|c|c|c|c|c|}
\hline $\begin{array}{l}\text { № } \\
\text { П } \\
/ \\
\text { п }\end{array}$ & $\begin{array}{l}\text { Ecological } \\
\text { and } \\
\text { geographical } \\
\text { origin of the } \\
\text { variety }\end{array}$ & $\begin{array}{l}\mathrm{Nu} \\
\mathrm{mbe} \\
\mathrm{r} \text { of } \\
\text { vari } \\
\text { eties }\end{array}$ & $\begin{array}{c}\text { Solu } \\
\text { ble } \\
\text { solid } \\
\text { s, \% }\end{array}$ & $\begin{array}{c}\text { Total } \\
\text { sugar } \\
, \%\end{array}$ & $\begin{array}{c}\text { Titrata } \\
\text { ble } \\
\text { acidity } \\
, \%\end{array}$ & Vitamin C, mg / $100 \mathrm{~g}$ & $\begin{array}{l}\text { Vitamin } \\
\text { P, mg / } \\
100 \mathrm{~g}\end{array}$ & $\begin{array}{l}\text { Anthocyanin } \\
\mathrm{s}, \mathrm{mg} / 100 \mathrm{~g}\end{array}$ \\
\hline \multirow{4}{*}{1} & $\begin{array}{l}\text { South of } \\
\text { Russia }\end{array}$ & \multirow{4}{*}{16} & & & & & & \\
\hline & $\begin{array}{l}\text { average }(\mathrm{M} \pm \\
\mathrm{m})\end{array}$ & & $\begin{array}{l}8,6 \\
\pm 0 \\
16\end{array}$ & $\begin{array}{l}6,6 \pm \\
0,11\end{array}$ & $\begin{array}{c}1,05 \\
\pm 0,0 \\
3\end{array}$ & $62,8 \pm 2,2$ & $84,9 \pm 3,9$ & $88,0 \pm 4,17$ \\
\hline & $\lim$ (variability) & & $\begin{array}{c}7,2 \\
- \\
9,7\end{array}$ & $\begin{array}{l}5,4- \\
7,7\end{array}$ & $\begin{array}{c}0,85- \\
1,28\end{array}$ & $45,5-74,6$ & $\begin{array}{l}58,3- \\
112,3\end{array}$ & $60,0-112,0$ \\
\hline & $\begin{array}{l}\text { coefficient of } \\
\text { variation }(\mathrm{V})\end{array}$ & & 7,4 & 7,8 & 13,4 & 13,6 & 17,9 & 18,3 \\
\hline \multirow{4}{*}{2} & $\begin{array}{l}\text { Central } \\
\text { Russia } \\
\end{array}$ & \multirow{4}{*}{4} & & & & & & \\
\hline & $\begin{array}{c}\text { average } \\
(\mathrm{M} \pm \mathrm{m})\end{array}$ & & $\begin{array}{l}8,5 \\
\pm 0 \\
33\end{array}$ & $\begin{array}{l}6,4 \pm \\
0,22\end{array}$ & $\begin{array}{c}0,95 \\
\pm 0,0 \\
7\end{array}$ & $55,0 \pm 5,32$ & $\begin{array}{c}84,3 \pm 5,7 \\
8\end{array}$ & $85,1 \pm 4,91$ \\
\hline & $\lim$ (variability) & & $\begin{array}{c}7,9 \\
- \\
9,5\end{array}$ & $\begin{array}{l}6,0- \\
7,2\end{array}$ & $\begin{array}{c}0,86- \\
1,15\end{array}$ & $39,5-63,2$ & $\begin{array}{l}70,0- \\
96,7\end{array}$ & $78,0-91,2$ \\
\hline & $\begin{array}{l}\text { coefficient of } \\
\text { variation (V) }\end{array}$ & & 7,7 & 7,8 & 14,9 & 19,4 & 13,7 & 9,8 \\
\hline \multirow{4}{*}{3} & England & \multirow{4}{*}{8} & & & & & & \\
\hline & $\begin{array}{l}\text { average }(M \pm \\
\mathrm{m})\end{array}$ & & $\begin{array}{l}8,5 \\
\pm 0, \\
22 \\
\end{array}$ & $\begin{array}{c}6,4 \pm \\
0,1\end{array}$ & $\begin{array}{c}1,05 \\
\pm 0,1 \\
7 \\
\end{array}$ & $59,8 \pm 3,57$ & $\begin{array}{c}95,2 \pm 4,8 \\
5\end{array}$ & $80,1 \pm 6,92$ \\
\hline & $\lim$ (variability) & & $\begin{array}{c}7,9 \\
- \\
9,5\end{array}$ & $\begin{array}{l}5,9- \\
7,2\end{array}$ & $\begin{array}{c}0,83- \\
1,19\end{array}$ & $44,8-70,1$ & $\begin{array}{l}73,6- \\
110,5\end{array}$ & $62,9-100,8$ \\
\hline & $\begin{array}{l}\text { coefficient of } \\
\text { variation }(\mathrm{V})\end{array}$ & & 6,9 & 7,1 & 25,5 & 15,8 & 13,5 & 21,1 \\
\hline \multirow{4}{*}{4} & Germany & \multirow{4}{*}{2} & & & & & & \\
\hline & $\begin{array}{l}\text { average }(\mathrm{M} \pm \\
\mathrm{m})\end{array}$ & & $\begin{array}{l}8,3 \\
\pm 0 \\
50\end{array}$ & $\begin{array}{l}6,3 \pm \\
0,30\end{array}$ & $\begin{array}{c}1,03 \\
\pm 0,1 \\
0\end{array}$ & $55,2 \pm 5,80$ & $\begin{array}{c}68,3 \pm 7,2 \\
0\end{array}$ & $84,1 \pm 5,9$ \\
\hline & $\lim$ (variability) & & $\begin{array}{c}8,0 \\
- \\
8,8 \\
\end{array}$ & $\begin{array}{l}6,1- \\
6,7\end{array}$ & $\begin{array}{l}1,01- \\
1,05\end{array}$ & $49,4-61,0$ & $\begin{array}{l}61,6- \\
75,5\end{array}$ & $80,2-90,0$ \\
\hline & $\begin{array}{l}\text { coefficient of } \\
\text { variation }(\mathrm{V})\end{array}$ & & 6,7 & 6,6 & 1,7 & 14,9 & 14,9 & 7,1 \\
\hline \multirow{2}{*}{5} & Netherlands & \multirow[b]{2}{*}{16} & & & & & & \\
\hline & average $(\mathrm{M} \pm$ & & 8,2 & $6,3 \pm$ & 0,93 & $61,0 \pm 2,35$ & $78,4 \pm 4,8$ & $80,7 \pm 3,74$ \\
\hline
\end{tabular}




\begin{tabular}{|c|c|c|c|c|c|c|c|c|}
\hline \multirow{3}{*}{ 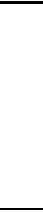 } & $\mathrm{m})$ & & $\begin{array}{c} \pm 0 \\
14\end{array}$ & 0,11 & $\begin{array}{c} \pm 0,0 \\
3\end{array}$ & & 0 & \\
\hline & $\lim$ (variability) & & $\begin{array}{c}7,5 \\
- \\
9,5\end{array}$ & $\begin{array}{l}5,7- \\
7,2\end{array}$ & $\begin{array}{l}0,70- \\
1,12\end{array}$ & $46,2-83,2$ & $\begin{array}{l}55,7- \\
117,5\end{array}$ & $60,0-108,0$ \\
\hline & $\begin{array}{l}\text { coefficient of } \\
\text { variation }(\mathrm{V})\end{array}$ & & 6,4 & 7,1 & 12,4 & 14,1 & 22,2 & 17,9 \\
\hline \multirow{4}{*}{6} & Italy & \multirow{4}{*}{17} & & & & & & \\
\hline & $\begin{array}{l}\text { average }(M \pm \\
\mathrm{m})\end{array}$ & & $\begin{array}{l}8,6 \\
\pm 0 \\
30\end{array}$ & $\begin{array}{l}6,6 \pm \\
0,11\end{array}$ & $\begin{array}{c}0,89 \\
\pm 0,0 \\
8\end{array}$ & $59,0 \pm 2,18$ & $82 \pm 4,13$ & $67,2 \pm 2,35$ \\
\hline & $\lim$ (variability) & & $\begin{array}{c}7,9 \\
- \\
9,3 \\
\end{array}$ & $\begin{array}{l}5,9- \\
6,9\end{array}$ & $\begin{array}{l}0,67- \\
1,07\end{array}$ & $50,6-67,2$ & $\begin{array}{l}41,8- \\
100,2\end{array}$ & $60,4-81,5$ \\
\hline & $\begin{array}{l}\text { coefficient of } \\
\text { variation }(\mathrm{V})\end{array}$ & & 6,4 & 6,8 & 11,1 & 9,2 & 19,7 & 8,5 \\
\hline \multirow{4}{*}{7} & Ukraine & \multirow{4}{*}{3} & & & & & & \\
\hline & $\begin{array}{l}\text { average }(M \pm \\
\mathrm{m})\end{array}$ & & $\begin{array}{l}8,5 \\
\pm 0, \\
38\end{array}$ & $\begin{array}{l}6,4 \pm \\
0,29\end{array}$ & $\begin{array}{c}1,01 \\
\pm 0,0 \\
8\end{array}$ & $60,1 \pm 2,58$ & $\begin{array}{c}81,3 \pm 5,4 \\
7\end{array}$ & $88,5 \pm 2,1$ \\
\hline & $\lim$ (variability) & & $\begin{array}{c}8,1 \\
- \\
9,0\end{array}$ & $\begin{array}{c}6,1- \\
6,8\end{array}$ & $\begin{array}{c}0,89- \\
1,08\end{array}$ & $58,9-63,7$ & $\begin{array}{l}76,0- \\
88,8\end{array}$ & $85,7-90,8$ \\
\hline & $\begin{array}{l}\text { coefficient of } \\
\text { variation }(\mathrm{V})\end{array}$ & & 7,9 & 7,9 & 13,5 & 6,5 & 11,6 & 3,7 \\
\hline \multirow{4}{*}{8} & Poland & \multirow{4}{*}{3} & & & & & & \\
\hline & $\begin{array}{l}\text { average }(M \pm \\
\mathrm{m})\end{array}$ & & $\begin{array}{c}8,1 \\
\pm 0 \\
49\end{array}$ & $\begin{array}{l}6,0 \pm \\
0,40\end{array}$ & $\begin{array}{c}0,87 \\
\pm 0,0 \\
8\end{array}$ & $61,1 \pm 12,1$ & $\begin{array}{c}75,3 \pm 12 \\
4\end{array}$ & $81,7 \pm 7,2$ \\
\hline & $\lim$ (variability) & & $\begin{array}{c}7,4 \\
- \\
8,5\end{array}$ & $\begin{array}{l}5,4- \\
6,3\end{array}$ & $\begin{array}{c}0,81- \\
1,0\end{array}$ & $47,6-78,6$ & $\begin{array}{l}60,4- \\
90,8\end{array}$ & $71,4-89,8$ \\
\hline & $\begin{array}{l}\text { coefficient of } \\
\text { variation }(\mathrm{V})\end{array}$ & & $\begin{array}{c}10 \\
6\end{array}$ & 11,7 & 10,3 & 36,7 & 26,7 & 12,2 \\
\hline \multirow{4}{*}{9} & USA & \multirow{4}{*}{19} & & & & & & \\
\hline & $\begin{array}{l}\text { average }(\mathrm{M} \pm \\
\mathrm{m})\end{array}$ & & $\begin{array}{c}8,5 \\
\pm 0, \\
15\end{array}$ & $\begin{array}{l}6,6 \pm \\
0,13\end{array}$ & $\begin{array}{c}0,91 \\
\pm 0,1 \\
0\end{array}$ & $59,1 \pm 2,35$ & $\begin{array}{c}100,6 \pm 4 \\
07\end{array}$ & $81,6 \pm 8,18$ \\
\hline & $\lim$ (variability) & & $\begin{array}{c}7,4 \\
- \\
10 \\
2\end{array}$ & $\begin{array}{l}5,7- \\
7,3\end{array}$ & $\begin{array}{l}0,74- \\
1,25\end{array}$ & $36,3-73,9$ & $\begin{array}{l}71,4- \\
143,6\end{array}$ & $57,7-115,9$ \\
\hline & $\begin{array}{l}\text { coefficient of } \\
\text { variation }(V)\end{array}$ & & 7,5 & 8,3 & 26,0 & 17,4 & 17,7 & 25,3 \\
\hline \multirow{4}{*}{$\begin{array}{l}1 \\
0\end{array}$} & France & \multirow{4}{*}{2} & & & & & & \\
\hline & $\begin{array}{l}\text { average }(M \pm \\
\mathrm{m})\end{array}$ & & $\begin{array}{l}8,4 \\
\pm 0, \\
30\end{array}$ & $\begin{array}{l}6,2 \pm \\
0,20\end{array}$ & $\begin{array}{c}1,01 \\
\pm 0,1 \\
0\end{array}$ & $66,5 \pm 0,14$ & $94,7 \pm 6,7$ & $83,9 \pm 5,14$ \\
\hline & $\lim$ (variability) & & $\begin{array}{c}8,2 \\
- \\
9,9\end{array}$ & $\begin{array}{c}5,8- \\
6,5\end{array}$ & $\begin{array}{l}0,92- \\
1,27\end{array}$ & $66,3-66,6$ & $\begin{array}{l}88,0- \\
101,4\end{array}$ & $69,0-98,8$ \\
\hline & $\begin{array}{l}\text { coefficient of } \\
\text { variation }(V)\end{array}$ & & 5,2 & 6,9 & 5,3 & 0,3 & 10,0 & 25,1 \\
\hline
\end{tabular}

Strawberry varieties of Ukrainian, English and American selection slightly differ in these indicators. On average, more than $1 \%$ of acids are contained in berries of a group of varieties of Russian, Ukrainian, German, French and English selection. The varieties of French selection $(66.5 \mathrm{mg} / 100 \mathrm{~g})$, as well as the selection of NCFSCHVW and Crimean EBS $(62.8 \mathrm{mg} / 100 \mathrm{~g})$ are distinguished by the level of accumulation of vitamin C. The greatest amount of vitamin $\mathrm{P}$ is characteristic of American varieties $(100.6 \mathrm{mg} / 100 \mathrm{~g})$, slightly lower than the English and French selection $(95.2$ and $94.7 \mathrm{mg} / 100 \mathrm{~g}$, respectively). It should be noted that in almost all groups, the coefficient of variation is 10 $20 \%$, except for the varieties of Polish (26.7\%) and Dutch selection (22.2\%). Varieties of Ukrainian selection ( $88.5 \mathrm{mg} / 100 \mathrm{~g}$ of anthocyanins), selection of NCFSCHVW and Crimean EBS (88.0 mg / $100 \mathrm{~g}$ ) are marked with bright colors of berries. The lowest 
variability in color of berries is in the varieties of Ukrainian, German and Italian selection. It should be noted that according to the complex of indicators (soluble solids, sugars, acidity, vitamin $\mathrm{C}$, anthocyanins), varieties of NCFSCHVW and Crimean EBS selection were distinguished.

Five groups of strawberry varieties were investigated according to the ripening period: early ripening ( 8 varieties), medium early (27 varieties), middle (36 varieties), medium late (9 varieties) and late (10 varieties). Comparison of strawberries of different ripening periods does not show clear differences between them in the chemical composition of berries (table 3). It has been established that varieties with an early ripening period accumulate slightly less soluble dry substances and sugars, while late-ripening varieties are distinguished by a higher content of soluble dry substances and sugars. The same pattern can be traced in the accumulation of vitamin $\mathrm{C}$ and $\mathrm{P}$. There were no clear differences in the acid content depending on the ripening time of the berries.

Table 3. The chemical composition of strawberries depending on the ripening time (average).

\begin{tabular}{|l|c|c|c|c|c|c|c|}
\hline $\begin{array}{c}\text { Ripening } \\
\text { period }\end{array}$ & $\begin{array}{c}\text { Number } \\
\text { of } \\
\text { varieties }\end{array}$ & $\begin{array}{c}\text { Soluble } \\
\text { solids, } \%\end{array}$ & $\begin{array}{c}\text { Total } \\
\text { sugar, } \\
\%\end{array}$ & $\begin{array}{c}\text { Titratable } \\
\text { acidity, } \%\end{array}$ & $\begin{array}{c}\text { Vitamin } \\
\text { C, mg/ } \\
100 \mathrm{~g}\end{array}$ & $\begin{array}{c}\text { Vitamin P, } \\
\mathrm{mg} / 100 \mathrm{~g}\end{array}$ & $\begin{array}{c}\text { Anthocyanins, } \\
\mathrm{mg} / 100 \mathrm{~g}\end{array}$ \\
\hline Early & 8 & $8,3 \pm 0,27$ & $\begin{array}{c}6,3 \pm 0,1 \\
8\end{array}$ & $\begin{array}{c}1,0 \pm 0,1 \\
2\end{array}$ & $\begin{array}{c}57,4 \pm 3,1 \\
1\end{array}$ & $78,8 \pm 4,19$ & $83,0 \pm 5,20$ \\
\hline $\begin{array}{l}\text { Medium } \\
\text { early }\end{array}$ & 27 & $8,5 \pm 0,48$ & $\begin{array}{c}6,5 \pm 0,2 \\
2\end{array}$ & $\begin{array}{c}0,84 \pm 0, \\
10\end{array}$ & $\begin{array}{c}58,8 \pm 3,7 \\
0\end{array}$ & $84,2 \pm 5,25$ & $74,7 \pm 5,87$ \\
\hline Middle & 36 & $8,4 \pm 0,35$ & $\begin{array}{c}6,4 \pm 0,2 \\
0\end{array}$ & $\begin{array}{c}1,06 \pm 0, \\
13\end{array}$ & $\begin{array}{c}57,5 \pm 4,2 \\
3\end{array}$ & $84,1 \pm 5,90$ & $83,2 \pm 4,95$ \\
\hline $\begin{array}{l}\text { Medium } \\
\text { late }\end{array}$ & 9 & $8,8 \pm 0,32$ & $\begin{array}{c}6,8 \pm 0,1 \\
7\end{array}$ & $\begin{array}{c}0,91 \pm 0, \\
09\end{array}$ & $\begin{array}{c}56,8 \pm 3,9 \\
0\end{array}$ & $85,7 \pm 4,30$ & $90,2 \pm 4,21$ \\
\hline Late & 10 & $8,8 \pm 0,29$ & $\begin{array}{c}6,8 \pm 0,2 \\
1\end{array}$ & $\begin{array}{c}0,93 \pm 0, \\
10\end{array}$ & $\begin{array}{c}65,6 \pm 5,1 \\
5\end{array}$ & $87,3 \pm 3,91$ & $82,3 \pm 3,99$ \\
\hline
\end{tabular}

The large variability of the chemical composition of berries over the years is associated with the extremely unstable nature of weather conditions during the growing season, which affects the accumulation of soluble solids, sugars, acids and vitamins. On the example of 13 varieties of strawberries, the results of studying the chemical composition and the degree of variability of a number of indicators are shown (table 4).

Table 4. Variability of the chemical composition of strawberries depending on varietal characteristics, 2012-2020.

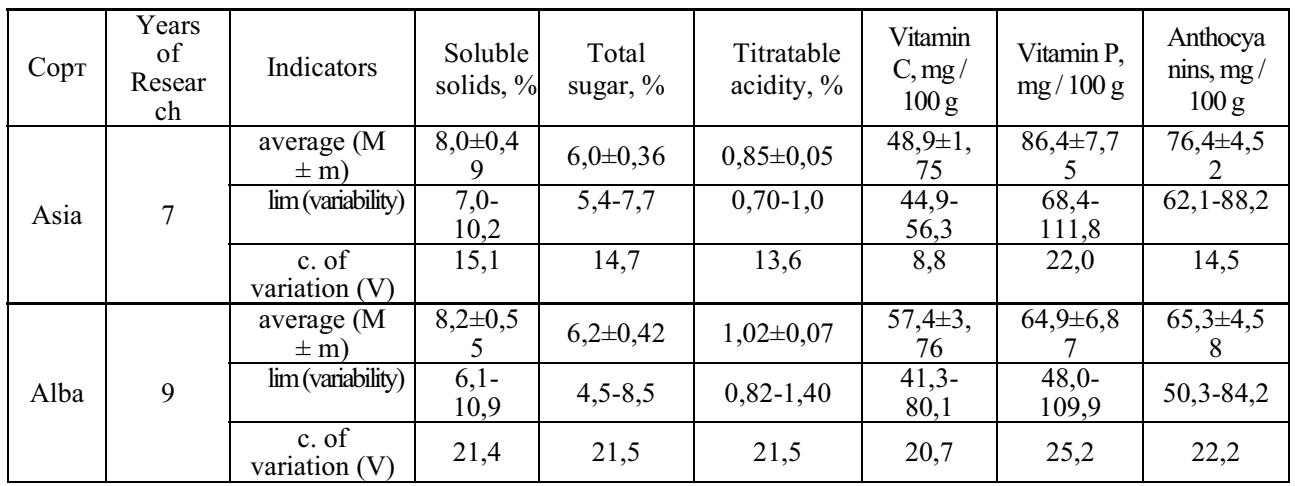




\begin{tabular}{|c|c|c|c|c|c|c|c|c|}
\hline \multirow{3}{*}{$\begin{array}{c}\text { Aros } \\
\mathrm{a}\end{array}$} & \multirow{3}{*}{7} & $\begin{array}{c}\text { average (M } \\
\pm \mathrm{m})\end{array}$ & $\begin{array}{c}8,6 \pm 0,5 \\
9 \\
\end{array}$ & $6,5 \pm 0,43$ & $0,89 \pm 0,09$ & $\begin{array}{c}62,8 \pm 2 \\
83 \\
\end{array}$ & $\begin{array}{c}100,2 \pm 7 \\
28\end{array}$ & $\begin{array}{c}78,9 \pm 4,9 \\
0 \\
\end{array}$ \\
\hline & & $\lim$ (variability) & $\begin{array}{l}7,0- \\
10,6 \\
\end{array}$ & $5,6-8,0$ & $0,69-1,16$ & $\begin{array}{c}55,4- \\
70,8 \\
\end{array}$ & $\begin{array}{l}72,0- \\
120,7 \\
\end{array}$ & $60,5-90,0$ \\
\hline & & $\begin{array}{c}\text { c. of } \\
\text { variation }(\mathrm{V})\end{array}$ & 16,8 & 16,0 & 21,4 & 11,1 & 17,8 & 15,2 \\
\hline \multirow{3}{*}{$\begin{array}{c}\text { Bogo } \\
\text { ta }\end{array}$} & \multirow{3}{*}{9} & $\begin{array}{l}\text { average (M } \\
\pm \mathrm{m})\end{array}$ & $\begin{array}{c}8,4 \pm 0,4 \\
0\end{array}$ & $6,4 \pm 0,29$ & $0,84 \pm 0,04$ & $\begin{array}{c}46,2 \pm 3 \\
69\end{array}$ & $\begin{array}{c}91,5 \pm 6,4 \\
3\end{array}$ & $\begin{array}{c}73,7 \pm 4,3 \\
7\end{array}$ \\
\hline & & lim(variability) & $\begin{array}{l}7,0- \\
12,9 \\
\end{array}$ & $5,6-9,7$ & $0,70-1,20$ & $\begin{array}{c}35,0- \\
76,6 \\
\end{array}$ & $\begin{array}{l}48,0- \\
116,0 \\
\end{array}$ & $\begin{array}{l}59,4- \\
115,5 \\
\end{array}$ \\
\hline & & $\begin{array}{c}\text { c. of } \\
\text { variation }(\mathrm{V})\end{array}$ & 17,6 & 16,8 & 18,6 & 29,8 & 26,3 & 22,2 \\
\hline \multirow{3}{*}{$\begin{array}{l}\text { Dars } \\
\text { elect }\end{array}$} & \multirow{3}{*}{8} & $\begin{array}{c}\text { average (M } \\
\pm \mathrm{m})\end{array}$ & $\begin{array}{c}9,9 \pm 0,5 \\
0\end{array}$ & $7,5 \pm 0,35$ & $0,92 \pm 0,06$ & $\begin{array}{c}66,6 \pm 2 \\
15\end{array}$ & $\begin{array}{c}101,4 \pm 7 \\
43\end{array}$ & $\begin{array}{c}69,0 \pm 4,3 \\
7\end{array}$ \\
\hline & & $\lim$ (variability) & $\begin{array}{l}8,6- \\
12,5 \\
\end{array}$ & $6,5-9,2$ & $0,60-1,19$ & $\begin{array}{c}61,2- \\
71,8 \\
\end{array}$ & $\begin{array}{l}73,1- \\
132,4 \\
\end{array}$ & $\begin{array}{l}56,1- \\
100,0 \\
\end{array}$ \\
\hline & & $\begin{array}{c}\text { c. of } \\
\text { variation }(\mathrm{V})\end{array}$ & 15,1 & 14,0 & 19,1 & 9,7 & 22,0 & 19,0 \\
\hline \multirow{3}{*}{$\begin{array}{c}\text { Cler } \\
\mathrm{y}\end{array}$} & \multirow{3}{*}{8} & $\begin{array}{l}\text { average (M } \\
\pm \mathrm{m})\end{array}$ & $\begin{array}{c}9,0 \pm 0,4 \\
3\end{array}$ & $6,6 \pm 0,26$ & $0,78 \pm 0,04$ & $\begin{array}{c}58,5 \pm 2 \\
82\end{array}$ & $\begin{array}{c}69,8 \pm 3,7 \\
3\end{array}$ & $\begin{array}{c}74,1 \pm 4,3 \\
7\end{array}$ \\
\hline & & $\lim$ (variability) & $\begin{array}{l}7,0- \\
11,7 \\
\end{array}$ & $5,3-8,4$ & $0,61-0,98$ & $\begin{array}{c}47,5- \\
75,7 \\
\end{array}$ & $48,0-82,0$ & $56,2-93,0$ \\
\hline & & $\begin{array}{c}\text { c. of } \\
\text { variation }(\mathrm{V})\end{array}$ & 16,1 & 13,1 & 15,0 & 15,6 & 17,7 & 17,4 \\
\hline \multirow{3}{*}{$\begin{array}{l}\text { M.Pa } \\
\text { ndor } \\
\text { e }\end{array}$} & \multirow{3}{*}{9} & $\begin{array}{c}\text { average (M } \\
\pm \mathrm{m})\end{array}$ & $\begin{array}{c}8,1 \pm 0,2 \\
3 \\
\end{array}$ & $6,1 \pm 0,17$ & $0,98 \pm 0,07$ & $\begin{array}{c}65,1 \pm 2 \\
45\end{array}$ & $\begin{array}{c}95,8 \pm 5,6 \\
9 \\
\end{array}$ & $\begin{array}{c}88,9 \pm 4,4 \\
7 \\
\end{array}$ \\
\hline & & $\lim$ (variability) & $6,9-9,2$ & $5,2-7,0$ & $0,80-1,40$ & $\begin{array}{c}53,7- \\
78,3 \\
\end{array}$ & $\begin{array}{l}66,9- \\
126,0\end{array}$ & $\begin{array}{l}66,5- \\
102,8\end{array}$ \\
\hline & & $\begin{array}{c}\text { c. of } \\
\text { variation }(\mathrm{V})\end{array}$ & 8,9 & 9,0 & 21,2 & 11,9 & 18,8 & 15,9 \\
\hline \multirow{3}{*}{ Nellie } & \multirow{3}{*}{9} & $\begin{array}{c}\text { average (M } \\
\pm \mathrm{m})\end{array}$ & $\begin{array}{c}8,4 \pm 0,4 \\
2 \\
\end{array}$ & $6,4 \pm 0,31$ & $1,20 \pm 0,05$ & $\begin{array}{c}47,9 \pm 4 \\
1 \\
\end{array}$ & $\begin{array}{c}84,2 \pm 5,8 \\
7 \\
\end{array}$ & $\begin{array}{c}79,7 \pm 4,5 \\
5 \\
\end{array}$ \\
\hline & & lim(variability) & $\begin{array}{l}7,4- \\
11,5 \\
\end{array}$ & $5,6-8,7$ & $1,0-1,50$ & $\begin{array}{c}30,8- \\
70,4 \\
\end{array}$ & $\begin{array}{l}49,2- \\
111,8 \\
\end{array}$ & $\begin{array}{l}60,0- \\
102,0\end{array}$ \\
\hline & & $\begin{array}{c}\text { c. of } \\
\text { variation }(\mathrm{V})\end{array}$ & 15,6 & 15,5 & 12,2 & 27,0 & 21,9 & 18,1 \\
\hline \multirow{3}{*}{ Onda } & \multirow{3}{*}{9} & $\begin{array}{c}\text { average (M } \\
\pm \mathrm{m})\end{array}$ & $\begin{array}{c}8,3 \pm 0,5 \\
8 \\
\end{array}$ & $6,3 \pm 0,46$ & $0,90 \pm 0,06$ & $\begin{array}{c}60,3 \pm 3 \\
07 \\
\end{array}$ & $\begin{array}{c}67,4 \pm 4,9 \\
4 \\
\end{array}$ & $\begin{array}{c}64,0 \pm 1,7 \\
8 \\
\end{array}$ \\
\hline & & $\lim$ (variability) & $\begin{array}{l}6,5- \\
11,6 \\
\end{array}$ & $4,9-8,80$ & $0,72-1,0$ & $\begin{array}{l}54,4- \\
82,0 \\
\end{array}$ & $41,8-98,8$ & $56,2-72,2$ \\
\hline & & $\begin{array}{c}\text { c. of } \\
\text { variation }(V)\end{array}$ & 18,1 & 19,4 & 10,1 & 15,2 & 27,2 & 8,8 \\
\hline \multirow{3}{*}{$\begin{array}{l}\text { Roxa } \\
\text { nne }\end{array}$} & \multirow{3}{*}{7} & $\begin{array}{l}\text { average (M } \\
\pm \mathrm{m})\end{array}$ & $\begin{array}{c}7,9 \pm 0,4 \\
3 \\
\end{array}$ & $6,0 \pm 0,42$ & $0,89 \pm 0,05$ & $\begin{array}{c}52,5 \pm 2 \\
8 \\
\end{array}$ & $\begin{array}{c}99,5 \pm 9,2 \\
9 \\
\end{array}$ & $\begin{array}{c}70,6 \pm 6,0 \\
2 \\
\end{array}$ \\
\hline & & $\lim$ (variability) & $6,7-9,8$ & $5,0-7,4$ & $0,66-1,0$ & $\begin{array}{c}41,4- \\
60,8 \\
\end{array}$ & $\begin{array}{l}62,5- \\
126,0 \\
\end{array}$ & $52,7-93,0$ \\
\hline & & $\begin{array}{c}\text { c. of } \\
\text { variation }(\mathrm{V})\end{array}$ & 13,3 & 12,9 & 136,9 & 15,5 & 22,6 & 20,9 \\
\hline \multirow{3}{*}{$\begin{array}{l}\text { Flore } \\
\text { nce }\end{array}$} & \multirow{3}{*}{9} & $\begin{array}{c}\text { average (M } \\
\pm \mathrm{m})\end{array}$ & $\begin{array}{c}8,3 \pm 0,4 \\
4\end{array}$ & $6,3 \pm 0,33$ & $0,92 \pm 0,04$ & $\begin{array}{c}67,2 \pm 3 \\
82\end{array}$ & $\begin{array}{l}104,9 \pm 5 \\
25\end{array}$ & $\begin{array}{c}85,9 \pm 3,4 \\
2\end{array}$ \\
\hline & & $\lim$ (variability) & $\begin{array}{l}6,8- \\
11,0 \\
\end{array}$ & $5,1-8,3$ & $0,76-1,16$ & $\begin{array}{l}51,9- \\
82,7 \\
\end{array}$ & $\begin{array}{l}75,9- \\
126,0 \\
\end{array}$ & $71,7-98,9$ \\
\hline & & $\begin{array}{c}\text { c. of } \\
\text { variation }(\mathrm{V})\end{array}$ & 16,8 & 16,7 & 15,2 & 15,8 & 15,8 & 12,6 \\
\hline \multirow{3}{*}{$\begin{array}{c}\text { Hone } \\
\mathrm{y}\end{array}$} & \multirow{3}{*}{9} & $\begin{array}{c}\text { average (M } \\
\pm \mathrm{m})\end{array}$ & $\begin{array}{c}8,4 \pm 0,4 \\
3 \\
\end{array}$ & $6,2 \pm 0,34$ & $0,99 \pm 0,06$ & $\begin{array}{c}59,2 \pm 2 \\
85\end{array}$ & $\begin{array}{c}98,1 \pm 4,8 \\
7 \\
\end{array}$ & $\begin{array}{c}93,4 \pm 7,4 \\
4\end{array}$ \\
\hline & & $\lim$ (variability) & $\begin{array}{l}6,3- \\
11,9 \\
\end{array}$ & $4,7-9,0$ & $0,77-1,48$ & $\begin{array}{c}42,7- \\
87,1 \\
\end{array}$ & $\begin{array}{l}66,6- \\
136,2 \\
\end{array}$ & $\begin{array}{l}61,6- \\
107,0 \\
\end{array}$ \\
\hline & & $\begin{array}{c}\text { c. of } \\
\text { variation }(\mathrm{V})\end{array}$ & 19,2 & 20,5 & 21,3 & 18,0 & 18,6 & 29,3 \\
\hline \multirow{3}{*}{$\begin{array}{l}\text { Elsa } \\
\text { nta }\end{array}$} & \multirow{3}{*}{9} & $\begin{array}{c}\text { average (M } \\
\pm \mathrm{m})\end{array}$ & $\begin{array}{c}8,4 \pm 0,2 \\
8\end{array}$ & $6,3 \pm 0,23$ & $0,82 \pm 0,02$ & $\begin{array}{c}61,0 \pm 3 \\
14\end{array}$ & $\begin{array}{c}91,3 \pm 5,1 \\
1 \\
\end{array}$ & $\begin{array}{c}78,4 \pm 4,0 \\
1 \\
\end{array}$ \\
\hline & & $\lim$ (variability) & $\begin{array}{l}6,0- \\
10,0 \\
\end{array}$ & $4,5-7,6$ & $0,67-0,92$ & $\begin{array}{c}38,7- \\
86,2 \\
\end{array}$ & $\begin{array}{l}66,4- \\
126,4 \\
\end{array}$ & $\begin{array}{l}53,8- \\
106,8 \\
\end{array}$ \\
\hline & & $\begin{array}{c}\text { c. of } \\
\text { variation }(\mathrm{V})\end{array}$ & 12,6 & 13,6 & 9,8 & 19,2 & 20,9 & 18,3 \\
\hline
\end{tabular}


For example, in 2017, in the 2-3 decades of May - early June, rains (precipitation 157.3 $\mathrm{mm}$, which is 2 times higher than the average long-term data), combined with the cool weather (the average temperature is $1.2^{\circ} \mathrm{C}$ lower than the average long-term data) negatively affected the accumulation of soluble solids and sugars in strawberries, which decreased by $9-13 \%$; acidity - increased by an average of $10 \%$. It was noted that a high coefficient of variation for these traits (15-20\%) in the varieties such as Darselect, Asia, Onda, Arosa, Florence, Bogota, Khoney, at the same time, insignificant variability is characteristic of the varieties Alba, Marmolada, Molling Pandora (V=6-9 \%).

Also, in the conditions of the Krasnodar Territory, the lack of rainfall against the background of extremely high temperatures in late May - early June negatively affected the accumulation of vitamins in berries. So in 2013, at the end of flowering - the beginning of ripening of strawberries (2-3 ten days of May), abnormally hot dry weather with a maximum average daily temperature of up to $32.1^{\circ} \mathrm{C}$ (average monthly temperature is 4.6 ${ }^{\circ} \mathrm{C}$ higher than the average long-term data) and no rain (by $80 \%$ below the average longterm data) did not allow the formation of high-quality of strawberries in terms of chemical composition. For example, the content of vitamin $\mathrm{C}$ was lower than the average in the varieties Alba (38.8 mg / $100 \mathrm{~g}$ ), Roxana (47.5 mg / $100 \mathrm{~g}$ ), Honey (52.8 mg / $100 \mathrm{~g}$ ), Onda $(47.5 \mathrm{mg} / 100 \mathrm{~g})$, Elsanta $(44.9 \mathrm{mg} / 100 \mathrm{~g})$. There was a high coefficient of variation for vitamin $\mathrm{C}(\mathrm{V}=27-29.8 \%)$ in varieties Nelli, Bogota. An insignificant degree of data variation (V not more than 9.7\%) was noted in the varieties Asia, Darselekt.

In 2018 and 2020, a higher content of vitamin C was found in strawberries (Alba variety - 58.9-68.7 mg / 100 g, Florence - 69.5-73.7 mg / 100 g, Onda - 69.1-72 , 1 mg / 100 g), since the ripening process took place in more favorable weather conditions (average daily temperature $18.8-21.1^{\circ} \mathrm{C}$ and precipitation within the range of long-term average data).

Thus, as a result of the studies carried out on the biochemical assessment of the berries of 90 varieties of garden strawberries, varieties with an increased content of soluble solids, sugars, acids, pectin substances, vitamins $\mathrm{C}$ and $\mathrm{P}$, anthocyanins were identified, which makes it possible to identify the most valuable varieties from a nutritional point of view, and also purposefully use them in breeding work when obtaining new varieties.

\section{Acknowledgment}

The work was performed on the collection of plant genetic resources All Russian Institute of Plant Industry (VIR) Collections of Plant Genetic Resources within the framework of the state assignment of VIR (budget project No. 0662-2019-0004 "Collections of VIR of vegetatively propagated crops (potatoes, fruit, berry, decorative, grapes) and their wild relatives - study and rational use ") and state assignment NCFSCHVW (budget project No. 0689-2019-0009 "Reveal patterns the influence of physical, chemical, and biotechnological methods of influence on fruit and berry and vegetable raw materials and develop multivariate dynamic models of biochemical management, microbiological and technological processes in the creation of innovative resource-saving technologies for storage and processing of fruits, berries, and vegetables ").

The authors declare no conflicts of interest.

\section{References}

1. S.K. Kim, D.S. Kim, D.Y. Kim, C. Chun, Food Chemistry, 184, 196 (2015)

2. A. Nowicka, A. Kucharska, A. Sokol-Letowska, I. Fecka, Food Chemistry, 270, 32 (2019)

3. M.Yu. Akimov, Nutrition issues., 88(2), 64 (2019) 
4. T.G. Prichko, Bulletin of the Russian Academy of Agricultural Sciences., 6, 78 (2011)

5. J. Pennington, R. Fisher, J. of Food Composition and Analysis, 23, 411 (2010)

6. M. Asensi-Fabado, S. Munne-Bosch, Trends in Plant Science, 15 (10), 582 (2010)

7. E.N. Sedova, T.P. Ogoltsova, Program and methodology of variety study of fruit, berry and nut crops, 160 (1999)

8. The program of the North Caucasus Center for the selection of fruit, berry, flower and ornamental crops and grapes for the period until 2030, 49 (2013)

9. I.M. Skurikhin, Tables of the chemical composition and caloric content of Russian food products, 276 (2007)

10. F.Giampieri, et al., Nutrition, 28 (1), 9 (2012)

11. I.V. Lukyanchuk, E.V. Zhbanova, Fruit growing, 29, 150 (2017)

12. Z.A. Avdeeva, Fruit and berry production in Russia, 17, 228 (2006)

13. O.I. Kamzolova, Fruit growing, 18, 105 (2006)

14. V.N. Kamzolova, Fruit and berry production in Russia, 45, 124 (2016)

15. V. V. Yakovenko, Fruit and berry production in Russia, 49, 383 (2017)

16. E.V. Zhbanova, Fruit growing, 27, 241 (2015) 